\title{
Qualité et usages de l'eau de la retenue d'eau de Kogbétohoue, dans la commune d'Aplahoue (Sud-Ouest, Bénin)
}

\author{
Coffi Justin Noumon ${ }^{1}$, Domiho Japhet Kodja ${ }^{2}$, Ernest Amoussou ${ }^{2}$, Luc O. Sintondji ${ }^{1}$, Daouda Mama ${ }^{3}$, \\ and Euloge K. Agbossou ${ }^{1}$ \\ ${ }^{1}$ Laboratoire d'Hydraulique et de Maîtrise de l'Eau (LHME), Institut National de l'Eau, Université d'Abomey \\ Calavi 01 BP 526 Cotonou, Bénin \\ ${ }^{2}$ Laboratoire Pierre Pagney Climat Eau Ecosystèmes et Développement, Faculté des Lettres Arts et Sciences \\ Humaines, Université d'Abomey Calavi 01 BP 526 Cotonou, Bénin \\ ${ }^{3}$ Laboratoire d'Hydrologie Appliquée (LHA), Institut National de l'Eau (INE), Université d'Abomey-Calavi, \\ 01 BP 526 Cotonou, Benin
}

Correspondence: Coffi Justin Noumon (justinoum2001@yahoo.fr)

Published: 16 November 2021

\begin{abstract}
Résumé. Le comblement et l'eutrophisation des retenues d'eau constituent des contraintes majeures pour les usages qu'en font les populations riveraines. L'étude bathymétrique a révélé une diminution moyenne de $0,116 \mathrm{~m} /$ an de la profondeur sur sept (07) ans. Ce niveau élevé de comblement s'explique par l'érosion des terres due à la dégradation du couvert végétal et le surpâturage. Les mesures directes de la qualité physique : le $\mathrm{pH}$, la température et l'oxygène dissous avec le multi paramètre PC HORIBA WATER QUALITY CHECKER U-10; les paramètres chimiques par la méthode chromatographique ICS 1000 et de dosage de la chlorophylle $a$ avec le spectrophotomètre DR 5000 par la Norme AFNOR T90-117 ont permis d'évaluer le risque d'eutrophisation par la grille de diagnostic d'Ifremer (2000). Les eaux sont dans un état eutrophe. Le comblement et l'eutrophisation de la retenue constituent des menaces potentielles à court terme pour la pisciculture, l'irrigation et la consommation tandis que la disponibilité quantitative de la ressource est menacée à moyen terme. Des relevés bathymétriques périodiques et des actions d'aménagement du bassin versant de la retenue sont nécessaires à la gestion intégrée de la retenue d'eau.
\end{abstract}

\section{Introduction}

L'eau est une ressource nécessaire à la vie, au développement et à l'environnement. L'eau douce, principal objet d'enjeux, ne constitue que $3 \%$ de la ressource mondiale dont les trois quarts sont stockés sous forme de glace (Perrin, 2000). La progression des dépôts solides engendre une perte de la capacité de stockage du réservoir, altère le rôle habituel des organes d'exploitation de l'ouvrage et raccourcit sa durée de vie (Bergahou et Ziadi, 1989).

Réalisée par le Projet d'Hydraulique Pastorale et Agricole (PHPA) en 2007, la retenue d'eau de Kogbétohouè vise essentiellement à : (i) réduire la vulnérabilité de la production agricole face aux aléas climatiques grâce à une meilleure maîtrise de l'eau; (ii) assurer l'approvisionnement en eau pour le bétail et (iii) susciter l'émergence d'autres activités telles que la pêche, la pisciculture, le maraîchage et la production de plants forestiers.

Les observations de terrain ont montré que la retenue d'eau de Kogbétohouè connaît un développement des macrophytes, signe d'eutrophisation qui peut affecter les usages (pisciculture, agriculture, abreuvage) auxquels elle se prête et que la pression agricole sur les terres du bassin contribue également à la dégradation du couvert végétal, source d'érosion et de transport solide de sédiment dans la retenue. Entre autres, les deux forages existants dans le village souffrent des difficultés de fonctionnement et ont été abandonnés par les populations 


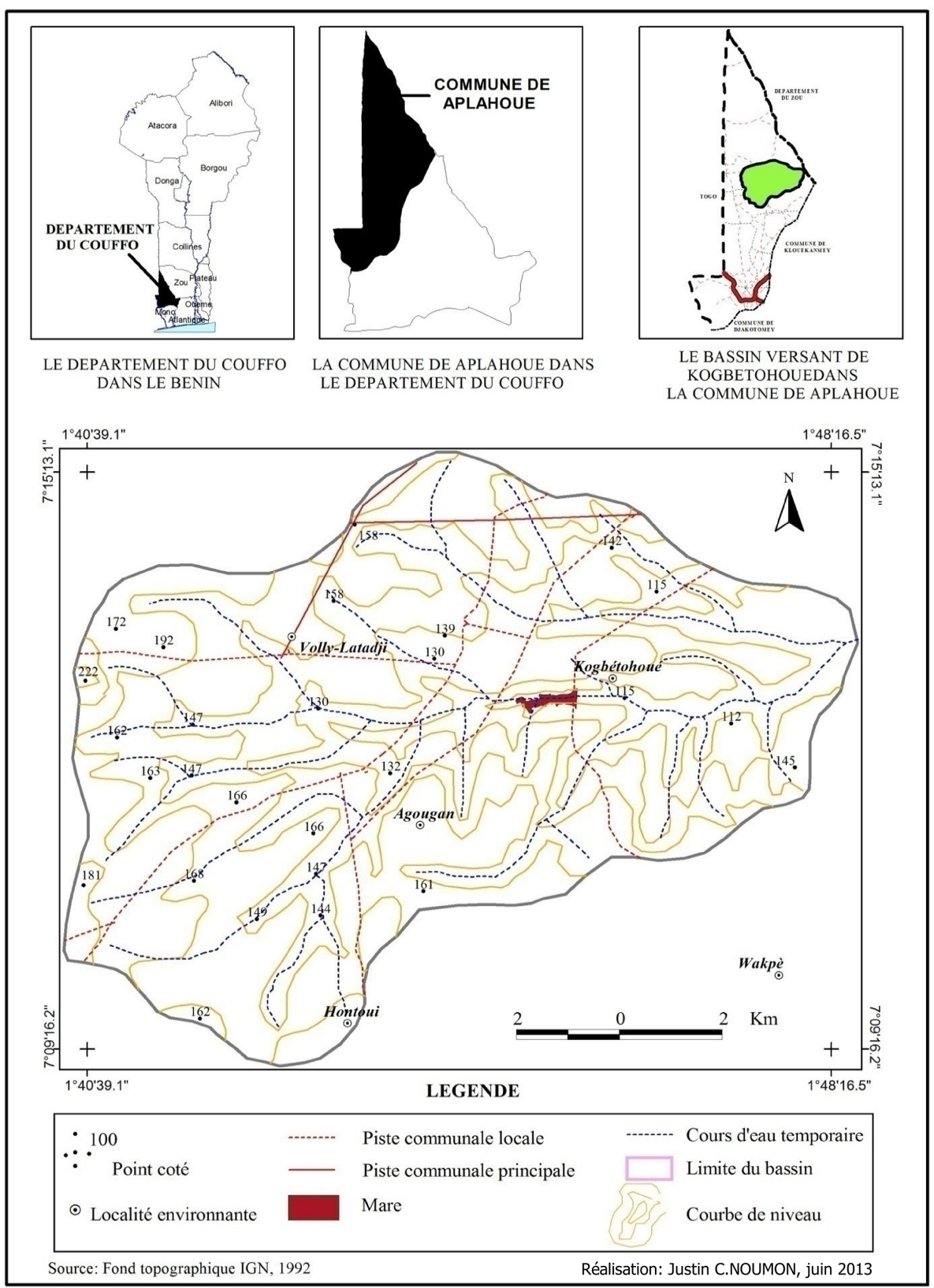

Figure 1. Carte de situation de la zone d'étude.

qui ont recours à l'eau de la retenue pour la consommation et les besoins domestiques.

Le comblement et l'eutrophisation des retenues d'eau ont été étudiés par plusieurs auteurs dont Mama (2010), Boukary (2012) et Ibouraïma et al. (2013) au Bénin. Ces travaux ont peu abordés les implications de ces deux phénomènes pour les usages de l'eau.

L'objectif de la présente étude est d'analyser les processus interdépendants du comblement et d'eutrophisation de la retenue d'eau de Kogbétohouè en relation avec les menaces à l'accès d'eau des populations riveraines.

\section{Matériels et methodes}

\subsection{Matériels d'étude}

La zone d'étude (Fig. 1), à l'instar de toute la commune d'Aplahoué (département du Couffo) qui abrite la retenue d'eau de Kogbétohouè, jouit d'un climat du type subéquatorial avec des précipitations annuelles moyennes de $1139 \mathrm{~mm}$ pour la période de 1983 à 2012 avec une moyenne des températures de l'ordre de $27^{\circ} \mathrm{C}$ et des écarts de thermiques assez faibles $\left(6^{\circ} \mathrm{C}\right)$. L'évapotranspiration potentielle annuelle est en moyenne de $1482 \mathrm{~mm}$ pour la même période Ecrire 
(Noumon, 2014). Le site de la retenue d'eau de Kogbétohouè est localisé au point de coordonnées géographique $07^{\circ} 12^{\prime} 44^{\prime \prime}$ Latitude Nord et $01^{\circ} 46^{\prime} 54^{\prime \prime}$ Longitude Est.

\subsection{Matériels de collecte}

Le matériel déployé pour la conduite de cette étude comporte essentiellement : la carte d'occupation du bassin versant de la retenue à partir des images satellitaires (Spot, Landsat de 1995, 2006 et 2012) pour estimer les superficies dégradées du couvert végétal à chacune de ces trois $(03)$ dates; le multi paramètre PC HORIBA WATER QUALITY CHECKER pour la mesure des paramètres physique de l'eau de la retenue;

les matériels (bouteilles en plastiques, des glacières) de prélèvements d'eau pour les analyses biochimiques de laboratoire, une (01) fiche d'enquête pour collecter les données des effectifs des différentes catégories d'animaux d'élevage et apprécier les charges pastorales (de l'aire d'influence de la retenue d'eau) et d'abreuvement (à la retenue d'eau). Sept (07) points d'échantillonnage sont définis de manière à avoir une bonne distribution spatiale des paramètres indicateurs de l'état trophique de la retenue (Fig. 2, annexe 1).

\subsection{Méthodes}

\subsubsection{Comblement de la cuvette}

Le tableau 1 résume les paramètres d'estimation du comblement de la cuvette.

Le traitement des images satellitaires avec le logiciel «ArcView » a permis d'apprécier l'évolution des unités d'occupation du sol en 1995, 2006 et 2012.

\subsubsection{Risque d'eutrophisation et état trophique}

Deux campagnes de prélèvements ont été effectuées (juin et septembre). Pour chaque campagne, sept (07) mesures et prélèvements sont faits aux emplacements définis (Fig. A1) La détermination des paramètres biochimiques caractéristiques de l'état trophique et le risque d'eutrophisation de la retenue a été faite par la méthode chromatographique ICS 1000 et le dosage de la chlorophylle $a$, a été effectué avec le spectrophotomètre DR 5000 par la Norme AFNOR T90-117. La détermination de l'état trophique de la retenue d'eau a été faite suivant la grille d'évaluation du risque d'eutrophisation de la colonne d'eau selon la méthode Ifremer (2000) complétée par le diagramme de classification du niveau trophique suivant l'échelle du MDDEP (2004). Pour chacun des sept (07) prélèvements, le risque d'eutrophisation a été apprécié pour chaque paramètre indicateur de l'état trophique. Ainsi, l'état global découlant de l'appréciation des différents paramètres a été donné pour les différents points échantillonnés et ceci pour les deux campagnes de prélèvements : juin (hautes eaux, pleine campagne vivrière, forte utilisation d'engrais NPK), septembre (petite saison des pluies).
Afin de déterminer le caractère oxydant ou réducteur de l'eau (Rejset, 2002) de la retenue d'eau, le pouvoir oxydant $\left(R_{H}\right)$ de l'eau a été évalué à partir de la formule suivante : $\mathrm{pH}=$ Potentiel en Hydrogène.

La valeur obtenue pour le pouvoir oxydant permet de situer l'eau dans les catégories suivantes selon Rejset (2002) :

- $R_{H} \geq 23$, le milieu est dit oxydant;

$-15<R_{H}<23$, le milieu est qualifié d'anoxique

- $R_{H}<15$, le milieu est dit réducteur

Les valeurs moyennes des différents paramètres ont permis de caractériser le pouvoir oxydant de l'eau de la retenue pour les deux campagnes de prélèvements.

\subsubsection{Qualité de l'eau et usages}

La qualité de l'eau a été appréciée en fonction des usages actuels de l'eau de la retenue (abreuvage, irrigation, aquaculture et consommation). Pour ce faire, quelques paramètres physico-chimiques indicateurs de l'aptitude de l'eau à ces usages selon le Système d'Evaluation de la Qualité de l'Eau des cours d'eau (SEQ-Eau, 2003) ont été utilisés.

Pour chaque paramètre, les résultats moyens des campagnes de prélèvements sont comparés aux valeurs limites des classes d'aptitude.

\section{Résultats}

\subsection{Facteurs de comblement de la retenue d'eau}

Le niveau annuel moyen de comblement de la retenue de 2007 à 2013 est estimé $0,116 \mathrm{~m} / \mathrm{an}$ (tableau 3). La dégradation du couvert végétal et la charge animale en sont les principaux facteurs responsables.

\subsubsection{Occupation du sol et état du couvert végétal}

Les unités d'occupation du sol sont les types de formations végétales (forêt, savanes, jachères, cultures) et des agglomérations. Les superficies des unités d'occupation du bassin versant en 1995, 2006 et 2012 et leur regroupement selon le degré d'anthropisation est résumé dans le tableau B1 (annexe). Les Forêts-Savanes sont passées de $49 \%$ en 1995 à $15 \%$ en 2012 tandis que les Champs-Jachères ont augmenté, passant de $49 \%$ à $83 \%$ pour la même période.

\subsubsection{Charge animale sur la retenue et les pâturage de son bassin versant}

La charge animale sur la retenue d'eau est estimée à 952,5 UBT contre une capacité de charge évaluée à 221 UBT (Noumon, 2015). Ceci montre qu'il s'agit d'un surpâturage : facteur de dégradation du couvert végétal avec l'érosion comme corollaire. 
Tableau 1. Paramètres d'estimation du comblement de la cuvette.

\begin{tabular}{ll}
\hline Profondeur maximale à la réalisation de la retenue en 2007 & $H_{\max 0(\mathrm{~m})}$ \\
\hline Profondeur maximale obtenue en 2013 & $H_{\max (\mathrm{m})}$ \\
\hline Age de la retenue & $A(\mathrm{ans})$ \\
Niveau de comblement pour la période & $H_{\max 0(\mathrm{~m})}-H_{\max (\mathrm{m})}$ \\
Comblement moyen annuel & $H=\left(H_{\max 0(\mathrm{~m})}-H_{\max (\mathrm{m})}\right) / A(\mathrm{~m} / \mathrm{an})$ \\
Durée de fonctionnement de la retenue d'eau & $H / H_{\max 0}$ \\
\hline
\end{tabular}

Tableau 2. Correspondance des valeurs des paramètres indicateurs de la qualité de l'eau.

\begin{tabular}{|l|l|l|l|l|l|}
\hline Couleur & Très bon & Bon & Moyen & Médiocre & Mauvais \\
\hline
\end{tabular}

\subsection{Qualité de l'eau}

\subsubsection{Pouvoir oxydant de l'eau}

La valeur obtenue pour le pouvoir oxydant (tableau 4 , annexe) est de 13 (inférieure à 15) aussi bien en juin qu'en septembre. Cette valeur montre que nous sommes en présence d'un milieu réducteur. En milieu réducteur, les nitrates et sulfates sont respectivement réduits en nitrites et sulfures. Ces deux dérivés sont de nature toxique à la vie aquatique notamment celle des poissons.

\subsubsection{Etat trophique et risque d'eutrophisation de la retenue d'eau}

Le niveau trophique de la retenue d'eau suivant le diagramme de classement du MDDEP (2004) est présenté dans les tableaux B3 et B4 (annexe). Le bilan général (tableau B5, annexe) donne des eaux hypereutrophes en Juin en période de pleine campagne de production agricole avec une utilisation des engrais NPK d'une part et eutrophes en septembre où l'intensification agricole est moindre.

\subsubsection{Conséquences du comblement et de l'eutrophisation de la retenue}

\section{Conséquences physico-économiques relatives au comblement de la retenue}

Le niveau annuel moyen de comblement de la retenue de estimé $0,116 \mathrm{~m} / \mathrm{an}$ entraîne une perte d'eau estimée à $1660 \mathrm{~m}^{3} / \mathrm{an}$ correspondant à une durée de fonctionnement de la retenue d'eau de 42 ans. Ceci constitue une menace réelle sur la disponibilité quantitative de l'eau de la retenue.

\subsubsection{Conséquences économiques et sanitaires liées à l'eutrophisation de la retenue d'eau}

Menaces sur la pisciculture

La concentration très élevée en phosphore (tableau 4) est la menace première sur la pisciculture en raison des effets secondaires notamment d'eutrophisation et son corollaire sur l'épuisement de l'oxygène.

\section{Menaces sur l'irrigation}

La concentration en microorganismes (tableau 5) suivie de la charge solide (environ $560 \mathrm{mg} / \mathrm{L}$ de matières sèches) constitue les menaces majeures sur l'usage de l'eau à des fins agricoles.

\subsubsection{Menaces sur l'abreuvement des animaux}

La qualité de l'eau ne présente pas de menace majeure à l'abreuvage (tableau 10).

\section{Menaces sur la production d'eau potable}

La forte teneur en coliformes totaux (tableau 9) et la turbidité (tableau 5) assez élevée constitue une contrainte pour l'aptitude de l'eau de la retenue à la production d'eau potable.

\section{Discussion et conclusion}

La dynamique de comblement $(0,116 \mathrm{~m} / \mathrm{an})$ est plus élevée que celle obtenu par les études de Mama (2010) sur le lac Nokoué au Bénin où la diminution de la profondeur est de l'ordre de $0,03 \mathrm{~m} / \mathrm{an}$ La durée de fonctionnement de la retenue de Kogbétohouè (42 ans) se retrouve bien dans la fourchette (12 à 114 ans) obtenues par Ibouraïma (2005) pour les retenues d'eau du département de l'Alibori au Bénin. L'évaluation du risque d'eutrophisation de la retenue d'eau de Kogbétohoué montre que les eaux sont dans un état général mauvais à médiocre vis-à-vis de leur état trophique (Noumon et al, 2015). Cette eutrophisation, caractéristique d'une pollution biologique du plan d'eau est marquée par la présence d'algues et le développement de plusieurs macrophytes. Elle 
Tableau 3. Niveau de comblement et durée de fonctionnement de la retenue.

\begin{tabular}{lrrrrr}
\hline $\begin{array}{l}\text { Paramètres d'estimation } \\
\text { du comblement }\end{array}$ & 2007 & 2013 & $\begin{array}{r}\text { Productivité de } \\
\text { sédiment }(\mathrm{m})\end{array}$ & $\begin{array}{r}\text { Apport annuel de } \\
\text { sédiment }(\mathrm{m})\end{array}$ & $\begin{array}{r}\text { Durée probable } \\
\text { de la retenue }\end{array}$ \\
\hline Profondeur max. (m) & 5,7 & 5,4 & 0,3 & 0,05 & 96 ans \\
Niveau d'eau à l'échelle (m) & 4,8 & 4,1 & 0,7 & 0,116 & 42 ans \\
\hline
\end{tabular}

Tableau 4. Aptitude de l'eau à la pisciculture en juin et septembre.

\begin{tabular}{|l|l|l|}
\hline Paramètres & $\begin{array}{l}\text { Aptitude en } \\
\text { Juin }\end{array}$ & $\begin{array}{l}\text { Aptitude en } \\
\text { Septembre }\end{array}$ \\
\hline $\begin{array}{l}\text { Oxygène dissous } \\
(\mathrm{mg} / \mathrm{L})\end{array}$ & & \\
\hline $\mathrm{PH}$ & & \\
\hline Ammonium $(\mathrm{mg} / \mathrm{L})$ & & \\
\hline Nitrite $(\mathrm{mg} / \mathrm{L})$ & & \\
\hline Nitrates $(\mathrm{mg} / \mathrm{L})$ & & \\
\hline MES $(\mathrm{mg} / \mathrm{L})$ & & \\
\hline $\begin{array}{l}\text { Phosphore Total } \\
(\mathrm{mg} / \mathrm{L})\end{array}$ & & \\
\hline
\end{tabular}

Tableau 5. Aptitude de l'eau à l'irrigation en novembre.

\begin{tabular}{|l|l|}
\hline Paramètres & $\begin{array}{l}\text { Aptitude en } \\
\text { Novembre }\end{array}$ \\
\hline $\begin{array}{l}\text { Résidus à sec à } 105^{\circ} \mathrm{C} \\
(\mathrm{mg} / \mathrm{L})\end{array}$ & \\
\hline Chlorures $(\mathrm{mg} / \mathrm{L})$ & \\
\hline $\begin{array}{l}\text { Coliformes totaux } \\
(\mathrm{u} / 100 \mathrm{ml})\end{array}$ & \\
\hline
\end{tabular}

résulte de l'altération de plusieurs caractéristiques physicochimiques de la qualité de l'eau notamment la transparence, l'oxygène dissous, la présence de l'azote, le phosphore et les sulfures à des concentrations élevées. L'eutrophisation constitue une menace sur la qualité des eaux pour les usages à des fins piscicoles (Chouti et al., 2010; Dèdjiho et al., 2013; Delhi et al., 2012; Francis-Lloyd, 2003). En effet, la prolifération des plantes aquatiques est à l'origine de la formation d'un écran en surface des eaux qui empêche l'oxygénation du milieu. De plus la décomposition de ces plantes, induit une augmentation de la quantité de matière organique dans le milieu dont la dégradation favorise l'asphyxie des espèces halieutiques (Mama et al., 2011). La production de sulfure et de nitrite (substances toxiques) inhibent le développement d'autres producteurs primaires et des poissons à grande sensibilité. Ceci peut provoquer un déséquilibre de la chaîne trophique (producteur/consommateur) d'un plan d'eau et entrâ̂ner des conséquences écologiques importantes (Dèdjiho et al, 2013). Mieux, Chambers et al, 2001 indiquaient que la présence des sulfures avec des concentrations de l'ordre de $10 \mathrm{mg} / \mathrm{L}$ est un signe précurseur de toxicité du milieu pour la vie aquatique, pour le bétail et la santé humaine.
Tableau 6. Aptitude de l'eau à l'abreuvage en juin et septembre.

\begin{tabular}{|l|l|l|}
\hline Paramètres & $\begin{array}{l}\text { Aptitude } \\
\text { en Juin }\end{array}$ & $\begin{array}{l}\text { Aptitude en } \\
\text { Septembre }\end{array}$ \\
\hline Nitrite $(\mathrm{mg} / \mathrm{L})$ & & \\
\hline Nitrates $(\mathrm{mg} / \mathrm{L})$ & & \\
\hline Sulfates & & \\
\hline Sulfures & & \\
\hline
\end{tabular}

En conclusion, l'étude de la qualité de l'eau de retenue de Kogbétohoué en relation avec ses usages a permis d'évaluer les menaces sur la ressource. Il ressort que les processus interdépendants du comblement et d'eutrophisation constituent des menaces réelles pour la pisciculture, l'irrigation et la consommation. Les conséquences physiques, économiques et sociales ressorties appellent à l'instauration d'une approche intégrée de la gestion de la retenue d'eau et de son bassin versant pour garantir les différents les usages que procure la retenue aux populations riveraines.

\section{Annexe A}

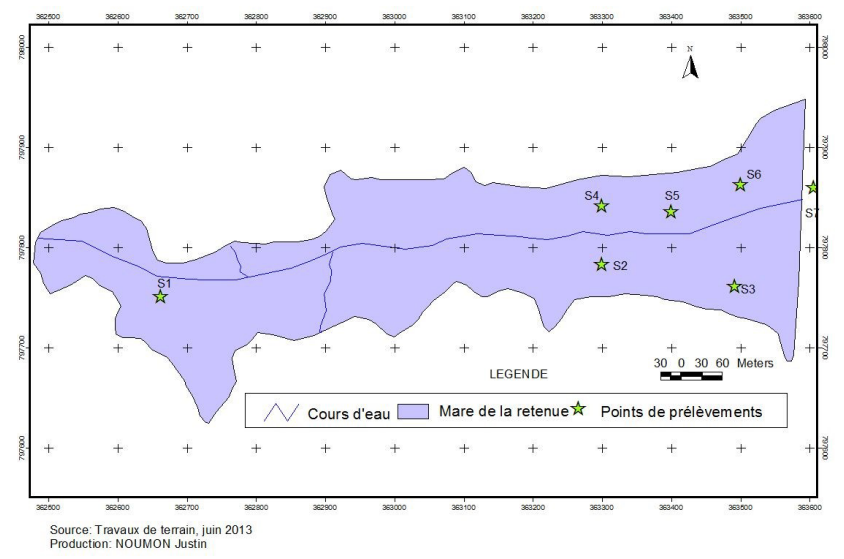

Figure A1. Carte de situation de points échantillons pour les analyses de qualité de l'eau. 


\section{Annexe B}

Tableau B1. Evolution des unités d'occupation du bassin versant selon les degrés d'anthropisation.

\begin{tabular}{lrrr}
\hline Unité d'occupation du sol & Superficie en 1995 (ha) & Superficie en 2006 (ha) & Superficie en 2012 (ha) \\
\hline Agglomération & $63,6(0,9 \%)$ & $84,7(1,20 \%)$ & $112,13(1,59 \%)$ \\
Forêts et Savanes & $3490(49,39 \%)$ & $1919(27,16 \%)$ & $1082(15,31 \%)$ \\
Champs et jachères & $3511,7(49,70)$ & $5061,6(71,64 \%)$ & $5871,2(83,1 \%)$ \\
\hline Total & $7065,3(100 \%)$ & $7065,3(100 \%)$ & $7065,3(100 \%)$ \\
\hline
\end{tabular}

Source : CENATEL : Images satellitaires du bassin versant de Kogbétohouè.

Tableau B2. Pouvoir oxydant de l'eau en juin et septembre.

\begin{tabular}{lrrrrl}
\hline Période & Eh & $T^{\circ}$ absolu & $\mathrm{pH}$ & $R_{H}$ & Nature de milieu \\
\hline Juin 2013 & $-35,57$ & 300,46 & 7,14 & 13,08 & Réducteur \\
Septembre 2013 & $-54,14$ & 300,48 & 7,42 & 13,02 & Réducteur \\
\hline
\end{tabular}

Tableau B3. Niveau trophique (MDDEP, 2004) de la retenue d'eau au mois de juin.

\begin{tabular}{|c|c|c|c|c|c|c|c|c|}
\hline Stations & 1 & 2 & 3 & 4 & 5 & S 6 & 7 & Moy./Ecartype \\
\hline Phosphore Total $(\mu \mathrm{g} / \mathrm{L})$ & 1600 & 1300 & 1100 & 1900 & 2800 & 800 & 2700 & $1766 / 772$ \\
\hline Chlorophylle $a(\mu \mathrm{g} / \mathrm{L})$ & 81 & 15,3 & 3,6 & 58,5 & 36 & 15,3 & 93,6 & $43,3 / 35$ \\
\hline Transparence (m) & 0,4 & 0,43 & 0,5 & 0,6 & 0,55 & 0,48 & 0,55 & $0.50 / 0,07$ \\
\hline Etat trophique & Hyper eutrophe & Eutrophe & Mésotrophe & Hyper eutrophe & Hyper eutrophe & Eutrophe & Hyper eutrophe & Hyper eutrophe \\
\hline
\end{tabular}

Tableau B4. Niveau trophique (MDDEP, 2004) de la retenue d'eau au mois de septembre.

\begin{tabular}{|c|c|c|c|c|c|c|c|c|}
\hline Stations & 1 & 2 & 3 & 4 & 5 & 6 & 7 & Moy./Ecartype \\
\hline Phosphore Total $(\mu \mathrm{g} / \mathrm{L})$ & 300 & 150 & 270 & 180 & 210 & 290 & 120 & $217 / 71$ \\
\hline Chlorophylle $a(\mu \mathrm{g} / \mathrm{L})$ & 37,8 & 10,8 & 5,4 & 9 & 12,6 & 3,8 & 207 & $45,9 / 79$ \\
\hline Transparence (m) & 0,85 & 0,72 & 0,78 & 0,85 & 0,85 & 0,85 & 0,4 & $0,75 / 0,16$ \\
\hline Etat trophique & Hyper eutrophe & Eutrophe & Méso eutrophe & Eutrophe & Eutrophe & Mésotrophe & Hypereutrophe & Eutrophe \\
\hline
\end{tabular}

Tableau B5. Evaluation du risque d'eutrophisation de la retenue d'eau (Ifremer, 2000).

\begin{tabular}{|c|c|c|c|c|c|c|c|c|}
\hline Mois & P1 & P2 & P3 & P4 & P5 & P6 & P7 & Bilan général \\
\hline Juin & Mauvais & Médiocre & Moyen & Mauvais & Mauvais & Médiocre & Mauvais & Mauvais \\
\hline Septembre & Mauvais & Médiocre & Moyen & Médiocre & Médiocre & Moyen & Mauvais & Médiocre \\
\hline
\end{tabular}


Disponibilité des données. Les données collectées et celles générées par la présente recherche sont stockées sur un disque dur externe privé et sont également conservées en version numérique au Laboratoire de l'Hydraulique et de La Maîtrise de l'Institut National de l'Eau de l'Université d'Abomey-Calavi (Bénin).

Collaborateurs. DJK a participé à l'analyse des données et à la relecture de l'article. La phase de collecte des données de terrain et celle de documentation sur la retenue d'eau ont été facilitées par EA et le LOS. Enfin DM et EKA ont suivi et encadré cette étude.

Intérêts concurrents. The contact author has declared that neither they nor their co-authors have any competing interests.

Clause de non-responsabilité. Publisher's note : Copernicus Publications remains neutral with regard to jurisdictional claims in published maps and institutional affiliations.

Déclaration du numéro spécial. This article is part of the special issue "Hydrology of Large River Basins of Africa". It is a result of the 4th International Conference on the "Hydrology of the Great Rivers of Africa", Cotonou, Benin, 13-20 November 2021.

\section{Références}

Bergahou, M. et Ziadi, K. : Quantification de l'érosion a l'échelle du bassin versant de l'Oued El Koudgi. Ecole Supérieure des Ingénieurs de 1'Equipement Rural de Medjez El Bab (Tunisie), 9 pp. 1989.

Boukari, O. : Etude de l'eutrophisation de la retenue d'eau de l'Okpara à Kpassa (Parakou), Mémoire de Master Professionnel en Ecohydrologie, 85 pp, LHA/FAST/UAC (BENIN), 2012.

Chambers, P. A., Kent, R., Charlton, M. N., Guy, M., Gagnon, C., Roberts, E., Grove, E., et Foster, N. : Les éléments nutritifs et leurs effets sur l'environnement au Canada, Environnement Canada, 271 pp. 2001.

Chouti, W., Mama, D., et Alapini, F. : Études des variations spatiotemporelles de la pollution des eaux de la lagune de Porto-Novo (Sud-Bénin), Int. J. Biol. Chem. Sci., 4, 1017-1029, 2010.
Dèdjiho, C. A., Mama, D., Tomètin, L., Nougbodé, I., Chouti, W. Sohounhlouè, D. C. K., et Boukari, M. : Évaluation de la qualité physic-chimique de certains tributaires d'eaux uses du lac Ahémé au Bénin, J. Appl. Bioscie., 70, 5608-5616, 2013.

Delhi, R., Benzha, F., Hilali, A., Tahiri, M., Kaoukaya, A., Baidder, L., Rhinane, H., et Hangouet, J. P. : Caractérisation de la qualité des eaux de la retenue Sidi Mohamed Ben Abdellah sur l'oued Bouregreg, ScienceLib, Editions Mersenne, 4, 120401, 20 pp. 2012.

Francis-Floyd, R. : Dissolved Oxygen for fish production. Institute of Food and Agriculture Sciences, University of Florida, 3 pp., 2003.

Ibouraïma, S. : Comblement des retenues d'eau d'abreuvement en zone agropastorale soudano-sahélienne : dynamique, bilan et impact de la sédimentation intra-cuvette, Thèse de Doctorat (unique) en gestion de l'Environnement, Laboratoire de Sédimentologie, FAST/UAC, 221 pp., 2005.

Ibouraima, S., Oyédé, L. M., and Sinsin, B. A. : Contribution de la vase au comblement des retenues d'eau de Gogbèdè et Guéné dans le Département de l'Alibori au Nord-Est du Bénin, Bulletin de Recherche Agronomique du Bénin (BRAB) Numéro 73, Juin 2013, 1025-2355 et ISSN (online), 1840-7099, 2013.

Ifremer : Mise à jour d'indicateurs du niveau d'eutrophisation des milieux lagunaires méditerranéens - Rapport final-09-2000, Tome I - 236 pp., Tome II - 196 pp., 2000.

Mama, D., Aïna, M., Alassane, A., Chouti, W., Boukary, O. T., Deluchat V., Bowen, J., Afouda, A., et Baudu, M. : Caractérisation physico-chimique et évaluation du risque d'eutrophisation du lac Nokoué (Bénin), Int. J. Biol. Chem. Sci., 5, 2076-2093, 2011.

MDDEP-Ministère du Développement durable, de l'Environnement et des Parcs : Réseau de surveillance volontaire des lacs, Les méthodes Québec, ISBN 978-2-550-53364-1, 5 pp, 2004.

Noumon, C. J. : Analyse du comblement et de l'eutrophisation de la retenue d'eau de Kogbétohouè (commune d'Apahouè) et implications pour une exploitation durable, Mémoire de Master Professionnel en Ecohydrologie, 88 pp, LHA/FAST/UAC, 2014.

Noumon, C. J., Mama, D., Dedjiho, C. A., Agbossou, E., et Ibouraima, S. : Évaluation de la qualité physico-chimique et du risque d'eutrophisation de la retenue d'eau de Kogbétohouè (Sud-Bénin), J. Appl. Biosci., 85, 7848-7861, 2015.

Perrin, C. : Vers une amélioration d'un modèle global pluie-débit au travers d'une approche comparative, Thèse de doctorat de l'Institut National Polytechnique de Grenoble, 276 pp., 2000.

Rejset, F. : Analyse des eaux : aspects réglementaires et techniques, Centre Régional de documentation pédagogique d'Aquitaine, 89 pp., 2002.

SEQ Eau : Système d'évaluation de la qualité de l'eau des cours d'eau. Grilles évaluation, Version 2, 40 pp., 2003. 